\title{
Parametric study on stressed volume and its application to the quantification of rolling contact fatigue performance of heterogeneous material
}

\author{
Wanyou Yang ${ }^{1}$, Yanyan Huang ${ }^{1}$, Qinghua Zhou ${ }^{1,3, *}$, Jiaxu Wang $^{1,2}$, \\ Xiaoqing Jin $^{2}$, Leon M. Keer ${ }^{3}$
}

\begin{abstract}
This research explores the influence of distributed inhomogeneities and surface asperities on the stressed volume of heterogeneous material in contact with a sphere. The recently developed modeling method for solving rough-surface contact problems involving distributed inhomogeneities, incorporated with FFT algorithms and a mesh differential refinement scheme, is utilized. The effective stressed volume, quantitatively described by effective depth, length and width, is investigated to characterize the rolling-contact-fatigue influence zone in heterogeneous material. Furthermore, a parametric study is conducted for the influences of inhomogeneity distribution and surface roughness on volumetric stress integral in order to understand the rolling contact fatigue performance of particle-laden inhomogeneous materials.
\end{abstract}

Keywords: stressed volume, rolling contact fatigue, distributed inhomogeneities, surface asperity, volumetric stress integral 


\section{Introduction}

Rotating mechanical components, such as bearings, are widely used in mechanical transmission systems to allow rotary motion and support significant cyclic load. Rolling contact fatigue $(\mathrm{RCF})$ is a main failure mode for bearings and other rotating mechanical components. Many RCF empirical and analytical models have been developed and proposed over the past few decades [1]. The most representative models for predicting RCF life of bearings were proposed by Lundberg and Palmgren [2], Ioannides and Harris [3], Zaretsky [4], which can be categorized as probabilistic engineering models. On the contrary, the models presented in Refs. [5, 6] belong to deterministic research models developed based on physical principles and with taking into account the actual mechanics of failure process.

An important concept involved in probabilistic RCF models is stressed volume, whose root can be traced back to the research work by Weibull [7]. The material strength was related to the volume of the material subjected to stress through specific equation in his work. Lundberg and Palmgren [2] further gave the formula definitions for the stressed volumes for point and line contacts, respectively. The stressed volume $V$ was quantified by raceway contact diameter $d$, the length of the running track $l_{t}$ and the depth of the critical shear stress $z$, i.e., $V=d l_{t} z$, when Hertzian contact theory was applied. The model proposed by Lundberg and Palmgren became the basis of the first ISO standards for bearing life used in the industry [8] and has been extensively used. Nevertheless, Harris and Yu [9] pointed out that the stressed volume utilized by Lundberg and Palmgren to encompass rolling contact fatigue failures does not show the real zone of stress influence. The actual influenced depth should be much larger. Besides, the Lundberg-Palmgren model, as well as the stressed volume, were associated with the stress of a single point rather than the subsurface stress distribution. The integrated effect of subsurface 
stress distribution on fatigue endurance should be considered, as suggested by Ioannides and Harris [3]. Moreover, the above mentioned stressed volume can only be applied to rolling contact analysis of homogeneous materials.

Materials involved in contact behavior are traditionally assumed to be homogeneous. However, in engineering practice, a second-phase material with different properties can always be observed in the matrix. For instance, large nonmetallic inclusions may be introduced into metal components due to faulty manufactures; fibers, whiskers, or particles are designed to reinforce composite materials to achieve high strength and stiffness. Those second-phase materials, can be termed as inhomogeneities according to the definition of Mura [10], inevitably exist at arbitrary locations in many engineering materials. They can produce disturbances to the elastic field at both the local and global scales. Therefore, the determination of stressed volume for heterogeneous contact must be quantitatively examined in order to better understand the roles played by inhomogeneities in the RCF performance.

The modelling of the influence of inhomogeneities on the elastic field of materials was explored by many research works. The well-known equivalent inclusion method (EIM) proposed innovatively by Eshelby $[11,12]$ was widely used to solve heterogeneous stress field due to the existence of inhomogeneities distributed in a full-space [13-15]. The conventional EIM can only deal with ellipsoidal-shaped inhomogeneities in an infinite medium applied with a uniform load. Nevertheless, taking the advantage of the cuboid inclusion solution proposed by Chiu [16], the EIM can be extended to arbitrarily shaped inhomogeneities [17]. The EIM is further introduced to solve heterogeneous contact problems, which should be analyzed in a half space, with the superposition scheme based on the method of images [18]. Representative work utilizing the improved EIM includes the fast method proposed by Leroux et al. [19] to study the effects of 
spherical inclusions on the contact pressure distribution and subsurface stress field in an elastic half-space; an efficient approximate numerical method for exploring the influence of distributed non-interpenetrating inhomogeneities on the contact of inhomogeneous materials [20] and RCF life of composite [21]; an updated superposition approach enables higher computational efficiency for partial-slip contact involving materials embedded with inhomogeneities by Wang et al. [22]. The method of images may induce numerical truncation errors for near-surface inclusions. A direct method capable of solving the elastic fields caused by a cuboidal eigenstrain was proposed by Liu et al. [23], and the complete set of integral kernels to express the elastic field caused by eigenstrain was derived. Further, the solution method by Liu et al. [23] was introduced to our recent work $[24,25]$ to study the rough-surface contact problems involving distributed inhomogeneity with arbitrary shapes.

The RCF performance of an engineering material is greatly effected by arbitrarily distributed non-metallic inhomogeneities [26]. Moreover, the consideration of surface roughness surely increases the complexity when solving elastic field disturbance for heterogeneous materials. In our recent work [25], a mesh differential refinement scheme, consist of independent surface, source, and target computational domains, was developed to enhance computation efficiency and flexibility. After that, a numerical solution method, incorporating with 3D fast Fourier transform (FFT) techniques and the mesh differential refinement scheme, was proposed for solving the contact with a smooth or rough surface involving distributed inhomogeneities [24]. The method was then introduced to predict the RCF lives of composite materials taking the benefit of the dual-beam focus ion beam scanning electron microscopy (FIB/SEM) technique [27]. In the present study, however, the recently developed numerical method is employed to quantify the stressed volume for identifying the influence scope of contact loading on a heterogeneous 
material, which is critical for the computation domain determination with considering inhomogeneity effect. Further, the focus is given to the parametric study on the effect of distributed inhomogeneities and surface asperities on the volumetric stress integral of materials. The obtained result is expected to be beneficial to better understanding the influences of nonmetallic particles and surface characteristics on the RCF lives of rotating mechanical components.

\section{Analytical procedure}

The employed numerical solution method for predicting the RCF lives of heterogeneous material under a rolling contact load was developed in our recent work [27], which is briefly summarized here for clarity. The selected computation domain is numerically discretized firstly. The mesh differential refinement scheme [25], as demonstrated in Fig. 1, is integrated for the present solution method. The solution method is composed of two parts, i.e., numerical solution for rough-surface heterogeneous contact and RCF prediction model based on the computation result of the former part. Figure 1 gives the basic flowchart of the numerical solution. Four main steps are involved to determine the disturbance in the elastic field caused by subsurface inhomogeneities and surface asperities. They are:

(1) Solving the surface pressure for rough-surface contact. A system of contact equations and inequalities used for describing load balance, surface deflection, contact body gap and contact compatibility is solved for the elemental pressures on contact surface. The conjugate gradient method (CGM) [28] is employed for fast solutions based on homogeneous-contact assumption. Readers may find more details in Ref. [28].

(2) Solving the elastic field induced by surface pressure. FFT algorithms, including a 2D discrete convolution and fast Fourier transform (2D DC-FFT) algorithm and a 2D layer-by-layer 
FFT algorithm [29], are introduced to accelerate the computation of surface deflection and stress distribution beneath the surface, respectively. The influence coefficients relating surface deformation and subsurface stress to surface traction are demonstrated in detailed in Refs. [30] and [31].

(3) Determination of the eigenstrains distributed in equivalent inclusions. The numerical EIM developed in our recent work [24] is implemented on all elementary inhomogeneities under consideration to compute the eigenstrains within the inclusions, which can equivalently replace the influence caused by inhomogeneities. A iterative scheme with stress or strain components represented in terms of Dundurs parameters [32] is adopted to implement the numerical EIM.

(4) Calculating the surface displacement caused by eigenstrains. The explicit solutions for the surface deformation produced by subsurface equivalent inclusions are given in Ref. [33]. A 2D DC-FFT algorithm can be applied between the subsurface domain enclosing all the inclusions under consideration and surface computational area in a way of layer-by-layer. The obtained surface deflection are then used to update the contact surface geometry.

The above four steps constitute a loop, which stops when the eigenstrain-surface deformation interactive process gets convergent. The elastic field caused by distributed inhomogeneities and surface asperities can be solved by the direct analytical method proposed by Liu et al. [23]. Full 3D FFT algorithms can be incorporated to greatly improve the computation efficiency. 
Figure 1: Numerical discretization of computation domain and the mesh differential refinement scheme.

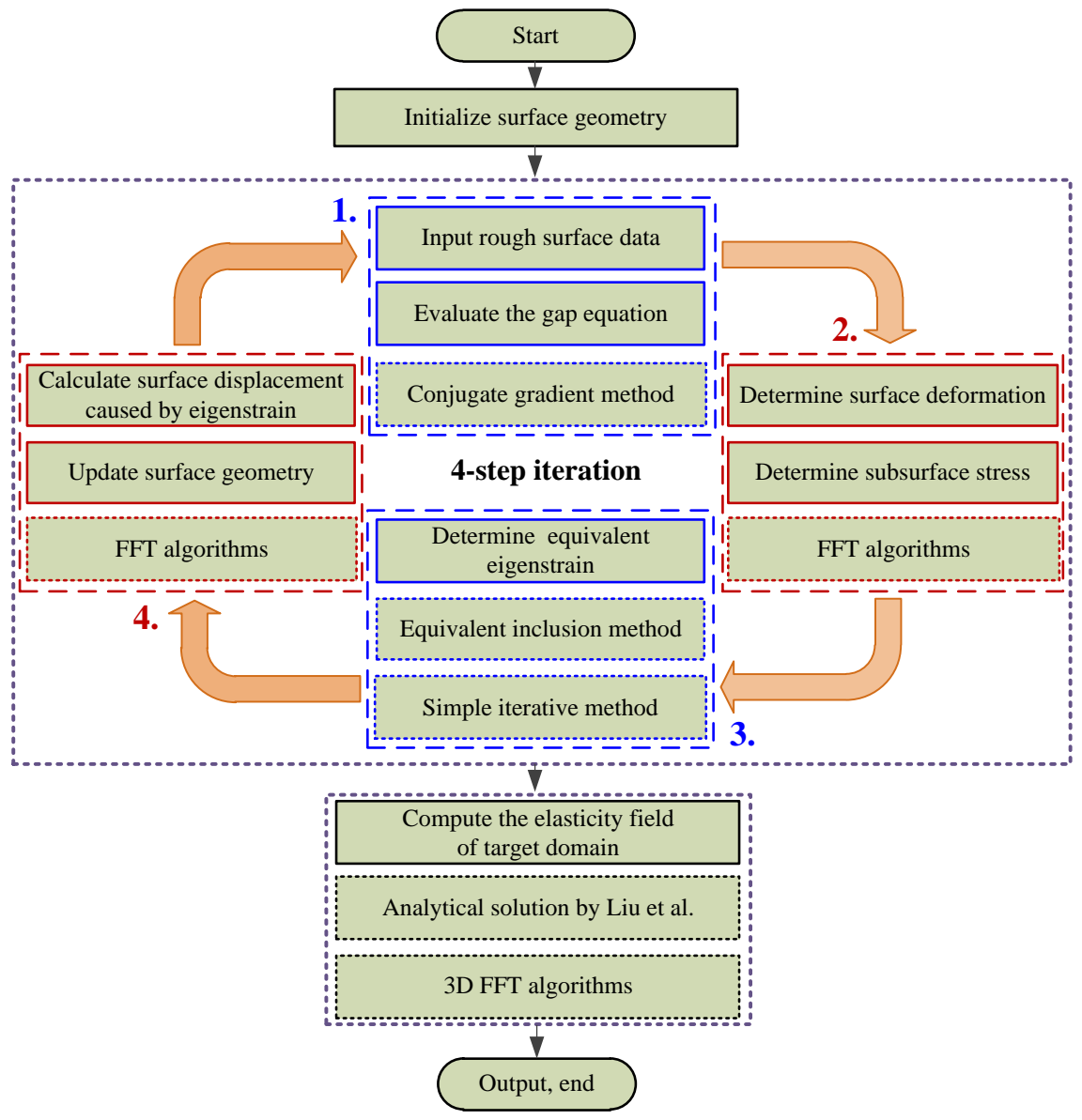

Figure 2: Flowchart of the unified numerical solution scheme for solving the elastic field of heterogeneous materials with rough surfaces under contact load. 
With the obtained elastic filed of heterogeneous contact, the RCF life prediction model developed by Zaretsky [4] is employed to predict the RCF lives of heterogeneous material applied with external contact loads. The model, as expressed in Eq. (1), correlates the number of loading cycles with the volumetric integral of equivalent stress over the computation domain

\[ \ln \frac{1}{S} \sim N^{e} \iiint_{V} \sigma_{e}^{e c} d V \]
where $S$ is the probability of survival, $N$ the contact fatigue life (number of stressed cycles), $V$ the stressed volume, $\sigma_{e}$ the equivalent stress, $e$ the Weibull slope and $c$ the stress exponent. It is worth noting that volumetric stress integral, $\operatorname{Vol}\left(\sigma_{\mathrm{e}}\right)=\iiint_{V} \sigma_{e}^{e c} d V$ is almost inversely proportional to the stressed cycle, $N$, when the survival probability $S=50 \%$ [34]. Therefore, $\operatorname{Vol}\left(\sigma_{\mathrm{e}}\right)$ can be utilized to qualitatively evaluate the approximate trend of material RCF lives. The capability of the present solution method on predicting the RCF lives of materials containing distributed inhomogeneities has been verified. The details about the verification of the method are given in Refs. [24, 25].

\section{Effective stressed volume for heterogeneous contact}

3.1 Definition of effective stressed volume for heterogeneous contact

The stressed volume of contact between a rigid sphere and a material with distributed cuboidal inhomogeneities is explored. The contact surfaces are assumed to be smooth first. Inhomogeneity volume fraction is set to be $5 \%$. A source domain of $2 a \times 2 a \times 4 a$ ( $a$ is the contact radius of the corresponding homogeneous contact case) is selected to enclose all the inhomogeneities under consideration, where a mesh of $128 \times 128 \times 256$ is applied on the source domain. All the cuboidal inhomogeneities have the same size with the side length 3 times that of 
a single discrete element of the source mesh, $\Delta x$. The surface domain is set as $2.25 a \times 2.25 a$ with the mesh size of $144 \times 144$. The target mesh is assumed to be identical to the source mesh in order to study the full stress field caused by the enclosed inhomogeneities. The location parameters, $x_{0}$, $y_{0}, z_{0}$, of cuboidal inhomogeneities (defined as the coordinates of inhomogeneity center) follow uniform distributions. A random number generation subroutine coded through Fortran [35] is used to determine the locations of arbitrarily distributed inhomogeneities. Specifically, $x_{0}, y_{0} \sim U(-a, a), z_{0} \sim U(0,4 a)$ ( $U$ represents uniform distribution), as shown in Fig. 3a.

The Young's moduli of the inhomogeneity and matrix are denoted by $E_{i}$ and $E_{m}$, respectively. Both stiff $\left(E_{i} / E_{m}=4.0\right)$ and compliant $\left(E_{i} / E_{m}=0.25\right)$ inhomogeneities are considered. The Poisson's ratios of the two materials, $v_{i}$ and $v_{m}$, are set as the same. The von Mises stress distributions in the $x-z$ plane solved by the present solution method are shown in Figs. 3b and 3c, which correspond to the stiff and compliant cases, respectively. All the stress values are normalized by the maximum von Mises stress $\sigma_{v m, \text { max }}^{h}$ yielded by homogeneous contact. The stress field of the matrix is greatly disturbed by those distributed inhomogeneities. Both stiff and compliant inhomogeneities increase the maximum von Mises stress. The relative values of the maximum stress, $\sigma_{v m, \text { max }} / \sigma_{v m, \text { max }}^{h}$, are 1.88 and 3.90, respectively. Compliant inhomogeneities cause more serious stress concentrations than stiff ones. Moreover, the elements with relatively larger stresses are concentrated around the area close to the surface applied with contact load. To some extent, the above subsurface stress distributions demonstrate that an inhomogeneity with a larger distance away from the loading area produces a weaker effect on the stress field of the matrix. In other words, a stressed volume exists for a material applied with any contact load on its surface. When an inhomogeneity is located out of the stressed volume, its influence on the elastic field of the matrix is negligible. 


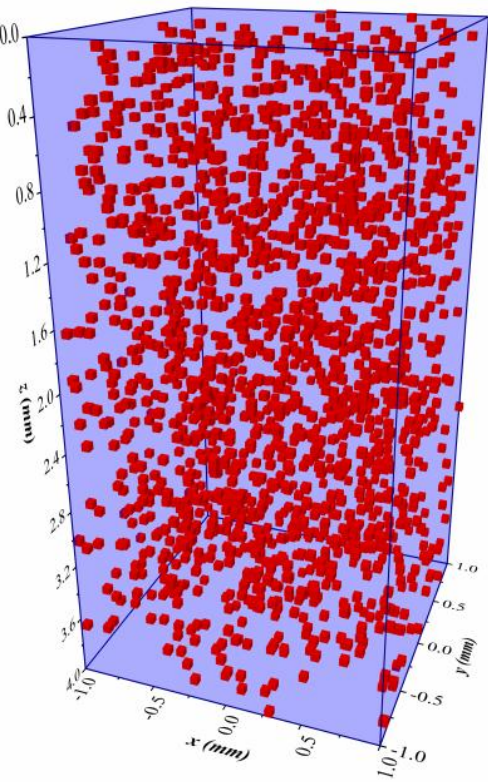

(a)

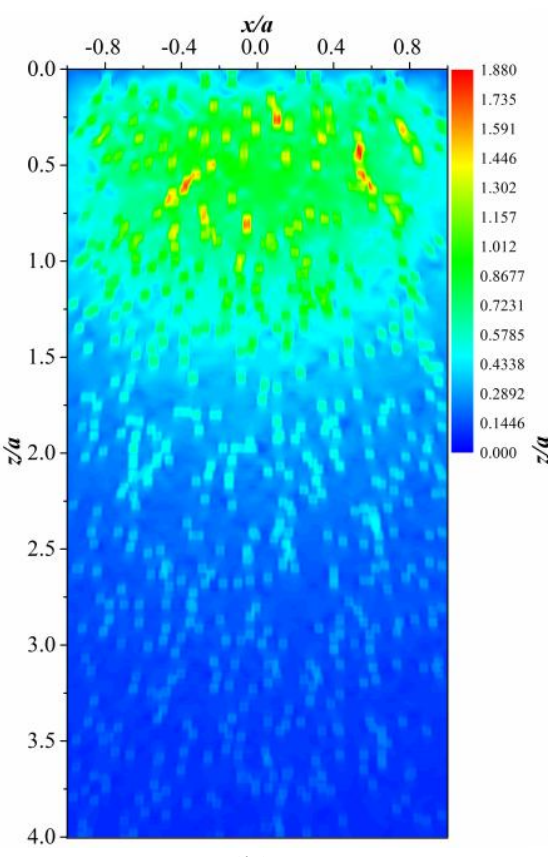

(b)

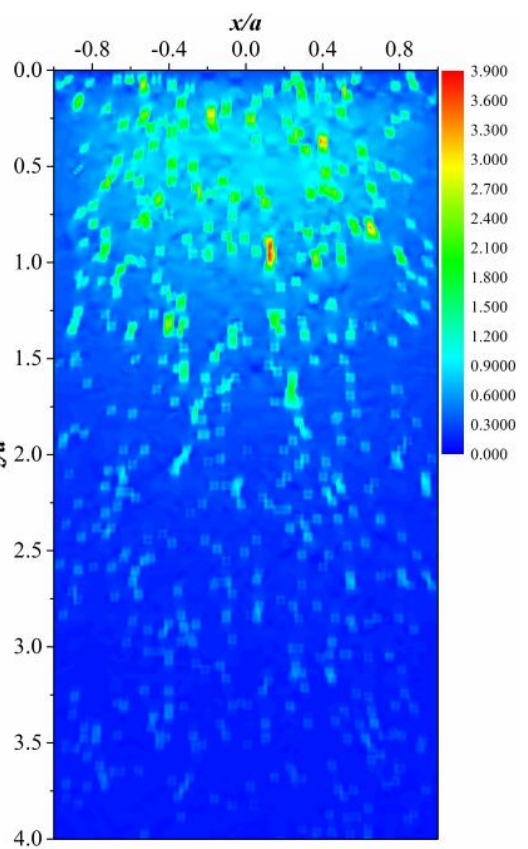

(c)

Figure 3: Heterogeneous contact involving a material with distributed cuboidal inhomogeneities of volume fraction $5 \%$.

a) Inhomogeneities following a uniform distribution. b) von Mises stress distribution in the $x-z$ plane for stiff $\left(E_{i} / E_{m}=4.0\right)$ inhomogeneities. c) von Mises stress distribution in the $x-z$ plane for compliant $\left(E_{i} / E_{m}=0.25\right)$ inhomogeneities.

$\mathrm{RCF}$ life is the most important factor to evaluate the performance of a material in engineering practice. As mentioned above, volumetric stress integral $\operatorname{Vol}\left(\sigma_{\mathrm{e}}\right)$ can be used to approximately quantify material RCF life. The above analyses prove that the influence of heterogeneous contact on the matrix elastic field is reduced with the increase in distance from the loading area. The effective stressed volume can therefore be utilized to characterize the RCF influence zone of heterogeneous contact, where all useful information about volumetric stress integral (or RCF) can be achieved. The stressed volume for heterogeneous contact in the present work is identified as a cuboidal domain quantified by effective depth, $H_{e}$ (along the $z$ direction), effective length, $L_{e}$ (along the $x$ direction), and effective width, $W_{e}$ (along the $y$ direction). The effective stressed volume can be set as the source or/and target domains to eliminate unnecessary computation burden when the present solution method is applied. 


$$
\operatorname{Vol}\left(\sigma_{e}\right)=\sum_{\gamma}^{N z} \sum_{\beta}^{N y} \sum_{\alpha}^{N x} \sigma_{e}^{e c}(\alpha, \beta, \gamma)
$$

where $N x, N y$ and $N z$ are mesh sizes along the $x, y, z$ directions for the target domain. According to Eq. (2), subdomain volumetric stress integral increases when involved computation domain (target domain) becomes larger. However, due to the existence of effective stressed volume, the integral can not always maintain growth at the same rate. The variation of the subsurface volumetric stress integral for the cases analyzed above is further studied quantitatively. von Mises stress is selected as the equivalent stress in Eq. (2), constants $c$ and $e$ are set to be 9 and 1.11, respectively [36]. Computational domains of $2 a \times 2 a \times 6 a$ and $4 a \times 2 a \times 2 a$ are selected as the reference fields for studying volumetric stress integral distributions along the depth $(z)$ and length $(x)$ directions, respectively. The deviation of the volumetric stress integral of a truncated computation domain with respect to that of the reference field is given as

$$
D_{s}=\operatorname{Vol}\left(\sigma_{e}\right) / \operatorname{Vol}\left(\sigma_{e}\right)_{\text {ref }} \times 100 \%
$$

where $\operatorname{Vol}\left(\sigma_{e}\right)_{\text {ref }}$ is the volumetric stress integral of the reference domain, $\operatorname{Vol}\left(\sigma_{e}\right)$ is that for a truncated domain. 


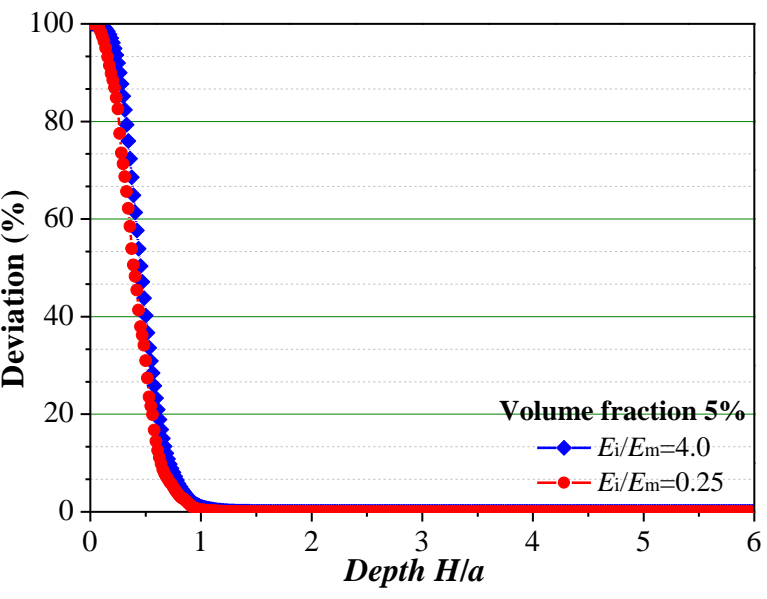

(a)

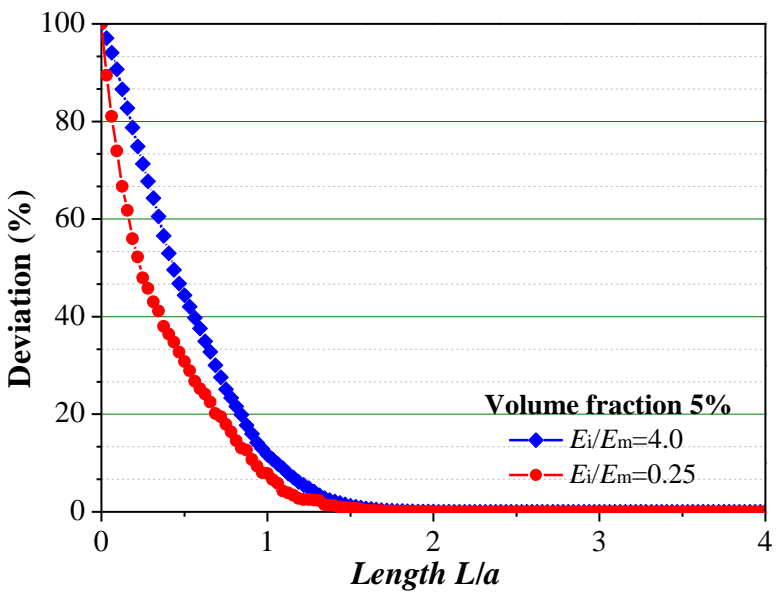

(b)

Figure 4: Volumetric stress integral deviations.

(a) Along depth direction (the $z$ direction) with reference computational domain of $2 a \times 2 a \times 6 a$. (b) Along length direction (the $x$ direction) with reference computational domain of $4 a \times 2 a \times 2 a$.

As shown in Fig. 4a, the depth of truncated domain changes from 0 to $6 a$. A mesh of $128 \times 128 \times 384$ is applied on the reference field, while that for the truncated domain is adjusted accordingly. When the truncated depth increases, the volumetric stress integral of the truncated domain increases rapidly. Comparing to stiff case, contact involving compliant inhomogeneities has relative smaller deviations. As shown in Fig. 3, compliant inhomogeneities induce higher stress concentrations than those by stiff ones. Volumetric stress integral is more sensitive to elements with larger stresses according to Eq. (2). Specifically, if $1 \%$ is selected as the threshold deviation to define the effective depth $H_{e}$ for volumetric stress integral of a contact body, i.e., the stress field deeper than $H_{e}$ contributes less than $1 \%$ to the whole volumetric stress integral, the effective depths are $1.02 a$ and $0.94 a$, respectively for the stiff and compliant cases in Fig. 3.

Moreover, in order to study the volumetric stress integral variation along the length $(x)$ direction, the reference field of $4 a \times 2 a \times 2 a$ is discretized into $256 \times 128 \times 128$ elements. The volumetric stress integral distribution along the width $(y)$ direction can be investigated in a similar way as that of the $x$ direction, which is therefore not discussed in the present work. The 
volumetric stress integral of the matrix increases quickly with the increase in the length of truncated domain. Smaller deviations are observed again for compliant cases than those yielded by stiff ones. The effective lengths for stiff and compliant cases are $1.56 a$ and $1.44 a$, respectively. The above results suggest that only the inhomogeneities located within a specific domain $\left(H_{e} \times L_{e} \times W_{e}\right)$, i.e., effective stressed volume, need to be considered when studying the influence of inhomogeneities on the RCF lives of heterogeneous materials.

\subsection{Determination of effective stressed volume for heterogeneous contact}

In order to analyze the influence of distributed inhomogeneities on the size of effective stressed volume quantitatively, more computations are conducted. The effective depth $H_{e}$ for volumetric stress integral of inhomogeneous contact is first investigated. The same model shown in Fig. 3a is utilized. Different material and distribution parameters of inhomogeneities are considered. The obtained effective depth $H_{e}$ with respect to the material difference between the inhomogeneity and matrix is shown in Fig. 5a. For stiff-inhomogeneity cases, inhomogeneity modulus is normalized by matrix modulus and the modulus ratio $E_{i} / E_{m}$ changes from 1 to 10 . For compliant cases, $E_{m} / E_{i}$ is set to be 1 to 10 , instead. Other parameters remain the same as those of Fig. 3a. For homogeneous case, the effective depth $H_{e}=1.08 a$. When multiple inhomogeneities are embedded in the matrix, the effective depth $H_{e}$ decreases for both stiff and compliant cases. When the material difference between the two materials enlarges, $H_{e}$ reduces due to the more serious stress concentrations arisen around the interfaces of the two materials. Moreover, the effective depth of stiff case is larger than that for compliant case when the material difference is the same. The obtained data are well fitted to two exponential functions, shown in Fig. 5a, for the two cases, respectively. 


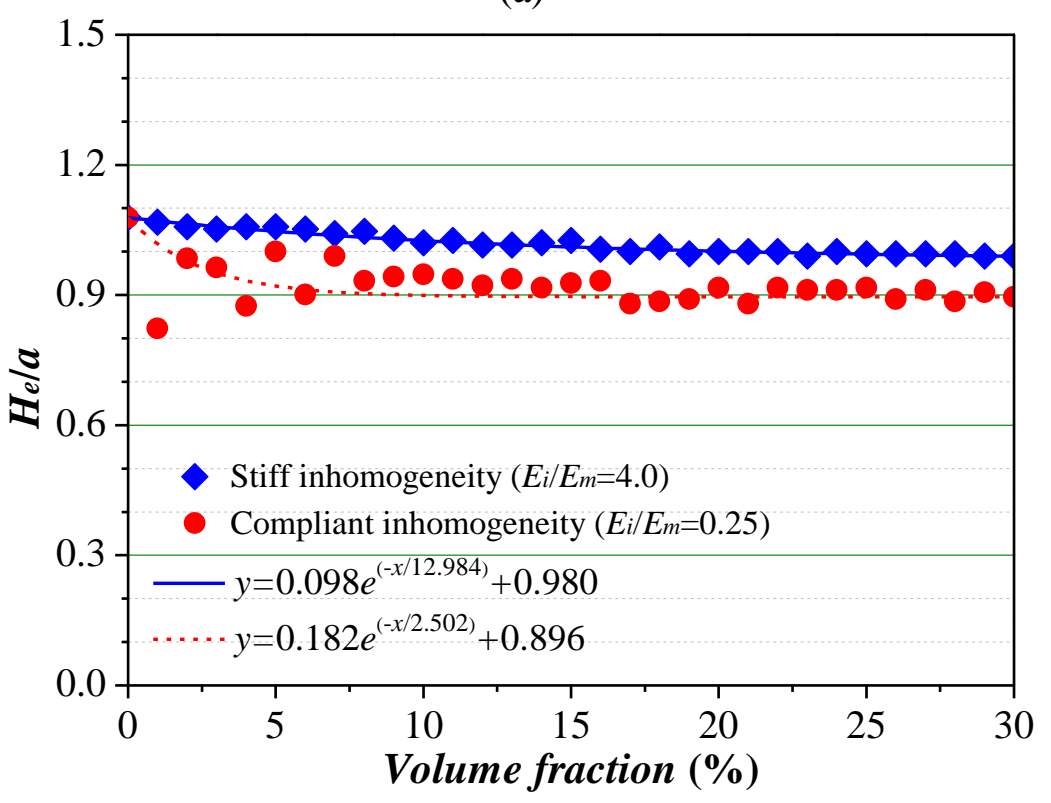

(b) 


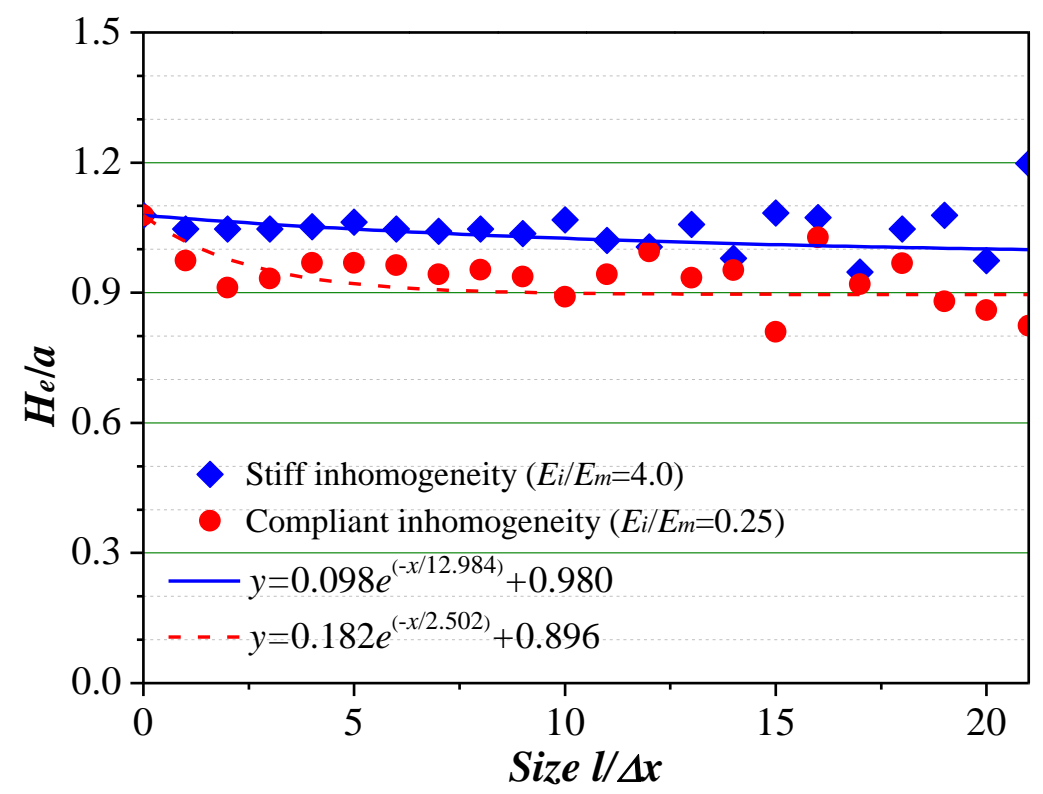

(c)

Figure 5: Effective depth $H_{e}$ for the volumetric stress integral of the inhomogeneous contact body. (a) Effective depth $H_{e}$ with respect to the material difference between the inhomogeneities and matrix. (b) Effective depth $H_{e}$ with respect to the volume fraction of inhomogeneities. (c) Effective depth $H_{e}$ with respect to the size of inhomogeneities.

Figure $5 \mathrm{~b}$ shows the obtained effective depth $H_{e}$ for inhomogeneities with different volume fractions. The volume fraction of distributed cuboidal inhomogeneities is changed from $0.0 \%$ to $30 \%$, while other parameters keep unaltered. An increase in volume fraction results in a decrease in effective depth. Again, the effective depth of stiff case is larger than that for compliant case when the two cases have identical volume fraction. The phenomenon is consistent with the result of the case involving material difference mentioned above.

The influence of inhomogeneity size on the effective depth is also studied. The volume fraction of inhomogeneity is $5 \%$. The side length of cuboidal inhomogeneities $l$ is change from 0.0 to $21 \Delta x$. Larger inhomogeneities are not considered because inhomogeneity number becomes too small to keep the statistical properties for inhomogeneity distribution. The results illustrated in Fig. 5c reveal that inhomogeneities with larger sizes produce smaller effective depths. 


\begin{abstract}
Moreover, the calculated depth values for both the stiff and compliant cases are more scattered due to the loss of statistics when inhomogeneities become larger in size.
\end{abstract}

In a similar way, the influence of inhomogeneity distribution parameters on the effective length $L_{e}$ of the stressed volume is investigated. The effective lengths with respect to the material properties, volume fraction, size of inhomogeneities are demonstrated in Figs. 6a, 6b and 6c, respectively. $L_{e}$ decreases with the increase in material difference, volume fraction and size of distributed inhomogeneities. The variation trends of the effective length caused by inhomogeneity material and distribution parameters are much similar to those of the effective depth. Exponential functions are also utilized to fit computation data and corresponding expressions are illustrated in Fig. 6.

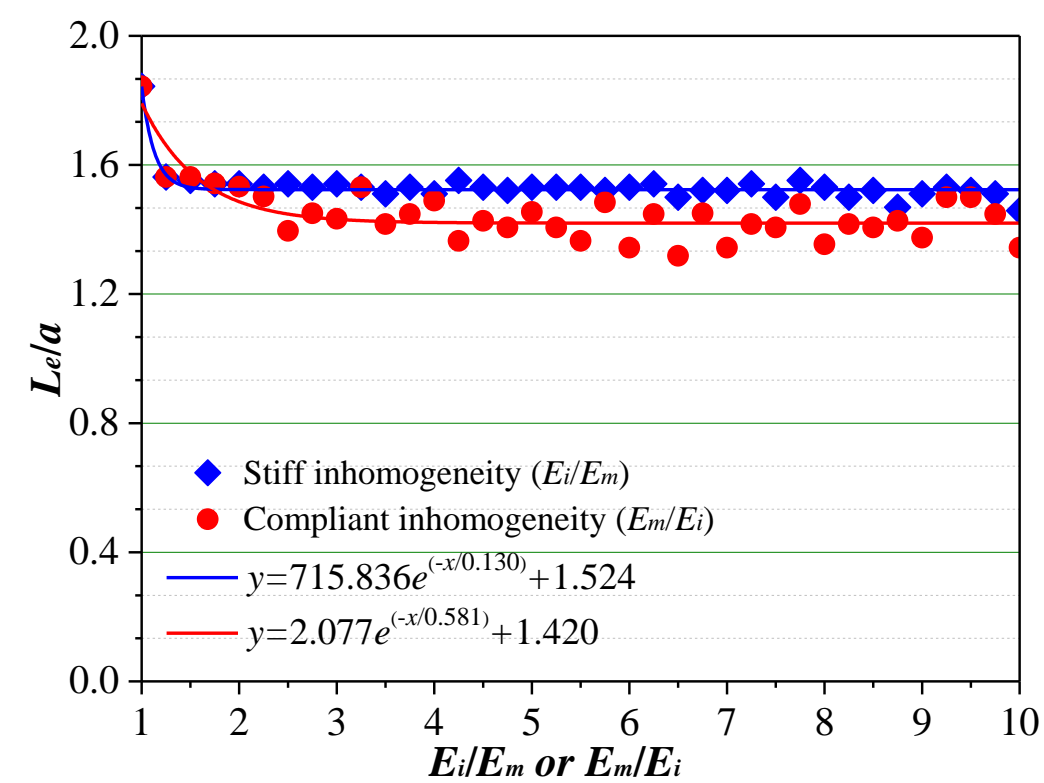

(a) 


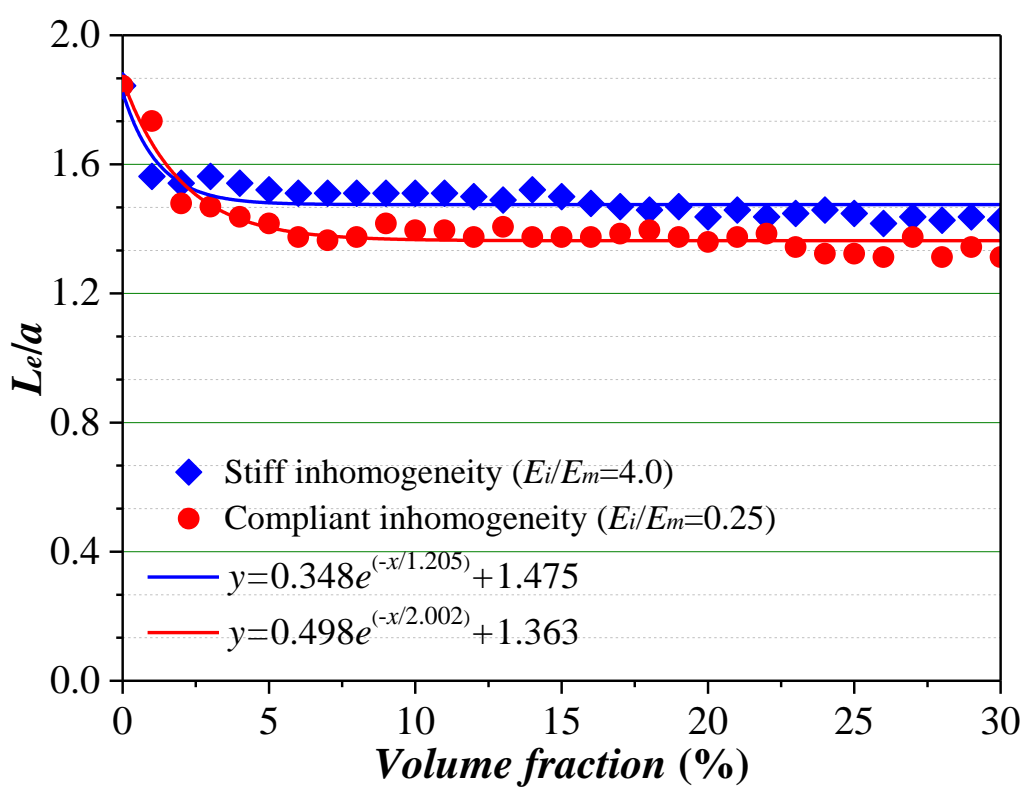

(b)

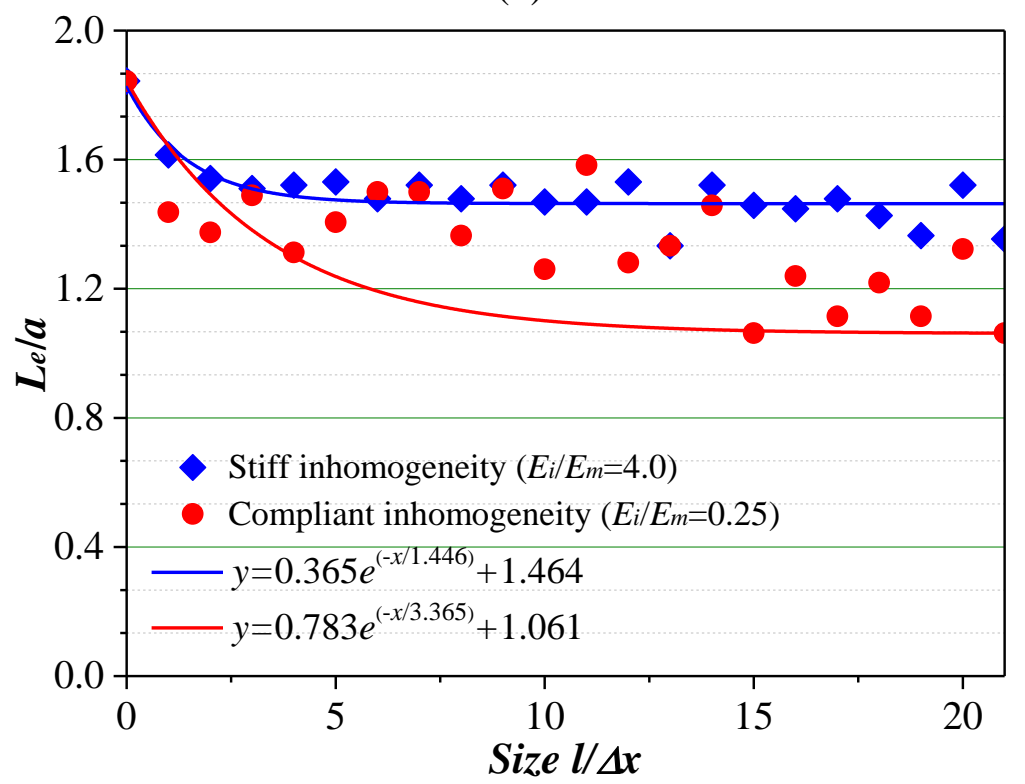

(c)

Figure 6: Effective length $L_{e}$ for the volumetric stress integral of the inhomogeneous contact body. (a) Effective length $L_{e}$ with respect to the material difference between the inhomogeneities and matrix. (b) Effective length $L_{e}$ with respect to the volume fraction of inhomogeneities. (c) Effective length $L_{e}$ with respect to the size of inhomogeneities.

The above analyses suggest that inhomogeneity properties and distribution parameters have remarkable influences on effective stressed volume. For instance, for a case with distributed inhomogeneities of volume fraction $10 \%, E_{i} / E_{m}=4.0, l=3 \Delta x$, the effective stressed volume is $1.02 a \times 1.51 a \times 1.51 a\left(H_{e} \times L_{e} \times W_{e}\right.$, effective width is assumed to be the same with effective length), 
which is much smaller than $2 a \times 2 a \times 2 a$ commonly adopted by conventional computations. The introduction of effective stressed volume brings a smaller computation domain when solving contact or RCF problem for heterogeneous materials. With the help of recently developed mesh differential refinement scheme [25], the concept of effective stressed volume is beneficial to not only save computation consumptions, but also simplify solution process for heterogeneous contact problem. More importantly, the above analyses can be followed as a guideline to determine the most economical computation domain when solving the elastic field of heterogeneous materials under an external load.

\section{Parametric study on volumetric stress integral}

The influence of distributed inhomogeneities and surface asperities on the volumetric stress integral of an inhomogeneous contact body is studied parametrically. The calculated volumetric stress integral is normalized by that of a corresponding homogenous case, $\operatorname{Vol}\left(\sigma_{\mathrm{e}}\right)_{\mathrm{H}}$.

$$
\operatorname{Vol}\left(\sigma_{e}\right)_{\mathrm{H}}=\sum_{\gamma}^{N z} \sum_{\beta}^{N y} \sum_{\alpha}^{N x} \sigma_{e}^{(h) e c}(\alpha, \beta, \gamma)
$$

where $\sigma_{e}^{(h)}$ is the equivalent stress for a homogenous case. The locations of the involved inhomogeneities in following computations follow uniformly random distributions.

\subsection{Material difference}

The presence of distributed inhomogeneities with two different material properties produces very different elastic fields, as shown in Figs. 3b and 3c. Therefore, it is necessary to investigate material difference effect on volumetric stress integral (RCF life) of heterogeneous materials. Cuboidal inhomogeneities with side length $l=3 \Delta x$ and volume ratio $5 \%$ are analyzed. For stiff or compliant inhomogeneity case, $E_{i} / E_{m}$ or $E_{m} / E_{i}$ changes from 1 to 10 . The surface mesh is 
$144 \times 144$ corresponding to a surface area of $2.25 a \times 2.25 a$. The source and target domains and meshes are adjusted accordingly based on the analysis results in Section 3.

The results are presented in Fig. 7a. A larger mismatch between the two materials leads to severe local stress concentrations produced by inhomogeneities beneath the surface, resulting in the increases of the achieved integral value for both the stiff and compliant cases. For cases with same material difference between the matrix and inhomogeneity, i.e., $E_{i} / E_{m}=E_{m} / E_{i}$, the obtained results for stiff and compliant cases are much different. Compliant-inhomogeneity groups produce relative higher integral values. When the material difference becomes larger, the volumetric stress integral induced by compliant inhomogeneities increases sharply. The result reveals that compliant inhomogeneities are greatly detrimental to RCF performance of materials under contact loads. Compliant inhomogeneity, such as pore, cavity, etc., must be avoided in engineering materials.

\subsection{Volume fraction}

The chosen inhomogeneities are equal-sized cuboids with side length $l=3 \Delta x$. Both stiff $\left(E_{i} / E_{m}=4.0\right)$ and compliant $\left(E_{i} / E_{m}=0.25\right)$ inhomogeneities are considered. The volume fraction varies from $0.0 \%$ to $30.0 \%$. Other contact conditions are the same with those in Fig. 3. The results are plotted in Fig. 7b. Both stiff and compliant inhomogeneities cause increases in volumetric stress integral, which suggests reductions in material RCF life. When volume fraction increases, i.e., more inhomogeneities of the same size are embedded in the domain, the integral value becomes larger, mainly due to stronger interactions between those inhomogeneities. Moreover, the integrals generated by distributed stiff inhomogeneities are found to be lower than those by compliant ones. As stated above, comparing to a stiff inhomogeneity, a compliant one 
may yield a larger stress concentration around it. Two power functions, shown in Fig. 7b, are employed to fit the obtained data for the compliant and stiff inhomogeneities, respectively.

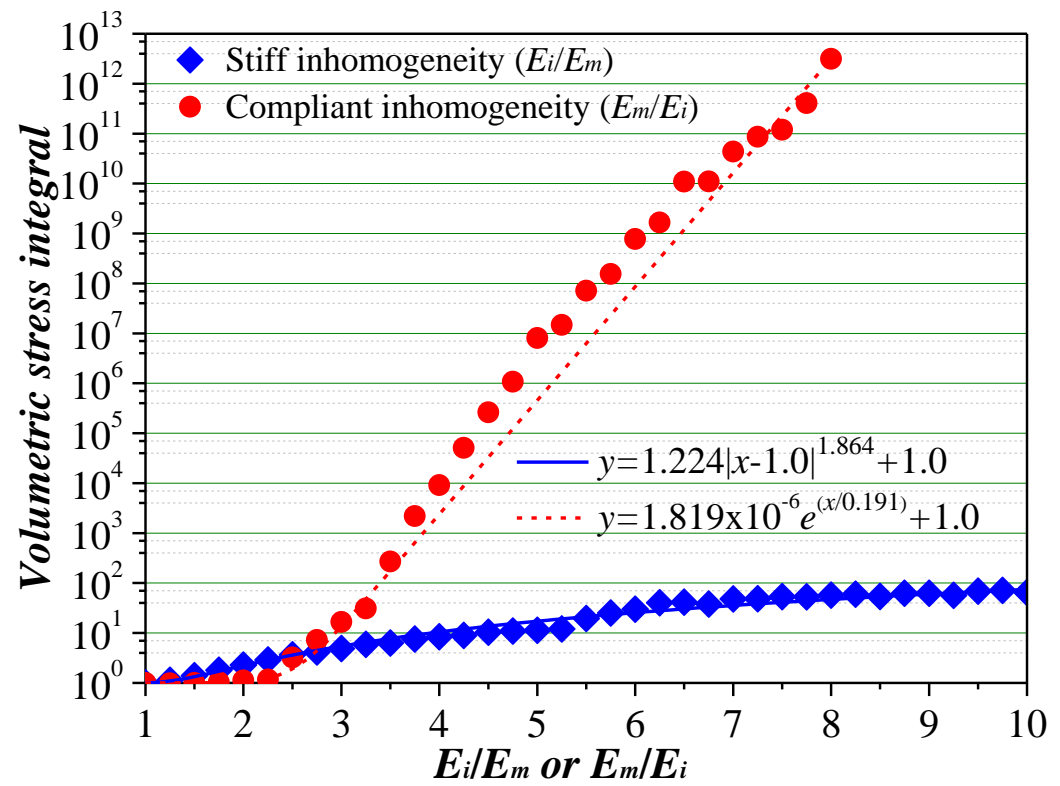

(a)

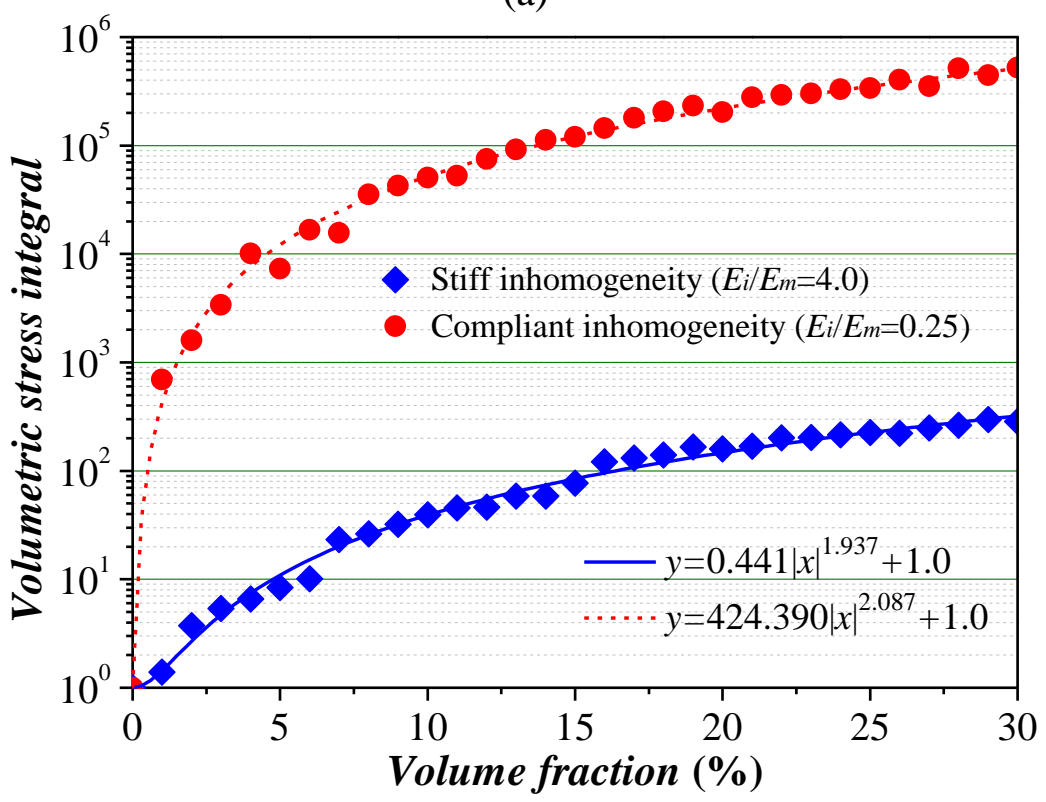

(b) 


\subsection{Inhomogeneity size}

The volume fraction of inhomogeneities is set as $5 \%$ with $E_{i} / E_{m}=4.0$ and 0.25 . Inhomogeneity with side length varying from 0 to $21 \Delta x$ is employed to study the effect of inhomogeneity size. As shown in Fig. 7c, similar to what observed in the above analyses, higher integral values are found for compliant inhomogeneities than those for stiff ones. Larger inhomogeneity size results in larger volumetric stress integrals of both the stiff and compliant cases. In other words, the RCF performance of heterogeneous material, such as composite materials, can be enhanced through refining the distributed inhomogeneities/reinforcements. 


\subsection{Inhomogeneity shape}

In the above parametric studies, the employed inhomogeneities are assumed to be cuboidal in all the cases. In this section, the influence of inhomogeneity shape on the volumetric stress integral of heterogeneous material is studied. The contact model in Fig. 3 is utilized again. Three kinds of shapes, i.e., cuboid, cylinder, sphere, are considered. For cylinder inhomogeneity, the two end planes are parallel to contact surface, and the diameter of end plane circles is assumed to have the equal size to its height. The radius of spherical inhomogeneity is $r$. The sizes of the cuboid and cylinder are selected accordingly to make sure that the three kinds of inhomogeneities have the same volume.

As shown in Figs. 8a and 8b, the volumetric stress integrals caused by inhomogeneities with different shapes are compared. Inhomogeneity volume fraction is set as $5 \%, r=10 \Delta x$. Inhomogeneities with different material properties $\left(E_{i} / E_{m}=1-10\right.$ (Fig. 8a) and $E_{m} / E_{i}=1-5$ (Fig. 8a)) are involved. The results demonstrate that compared to the other two kinds of inhomogeneities, spherically shaped inhomogeneity produces the smallest integral value, followed by cuboidal and cylinder inhomogeneities. The results suggest that compared with other shapes, sphere is conducive to the RCF performance of heterogeneous material to some extent. Inhomogeneities with different volume fractions $(0 \%-30 \%)$ and sizes $(r=5 \Delta x-30 \Delta x)$ are also taken into consideration. The results of volume fraction (Figs. 8c and 8d) and inhomogeneity size (Figs. 8e and 8f) confirm again that inhomogeneities of spherical shape yield smaller volumetric stress integrals than inhomogeneities with the other two shapes. 


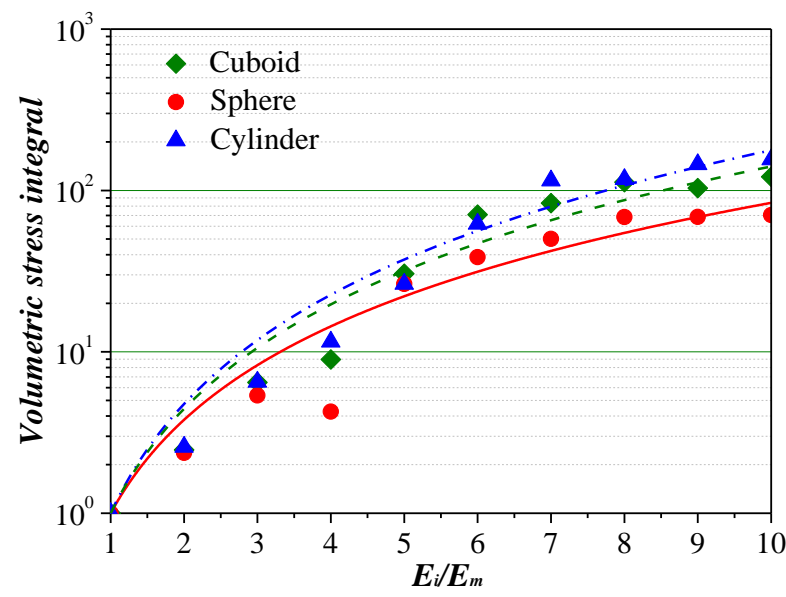

(a)

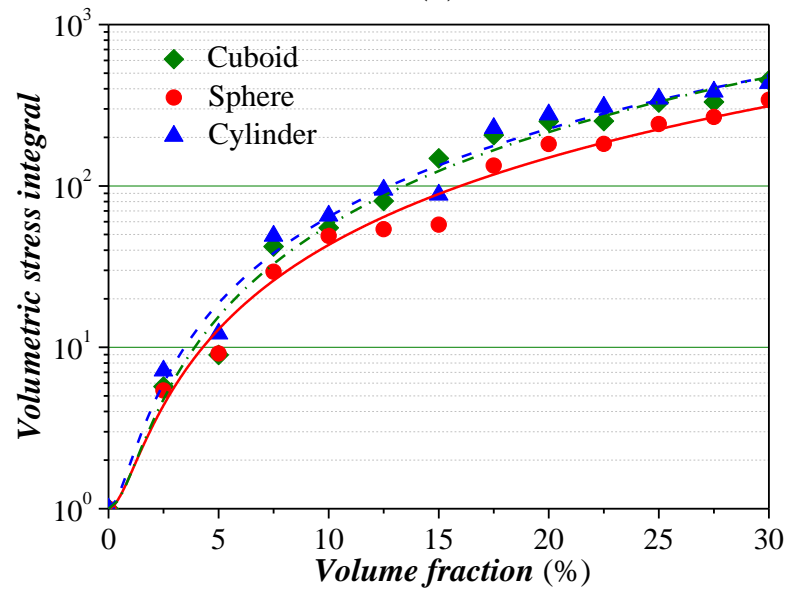

(c)

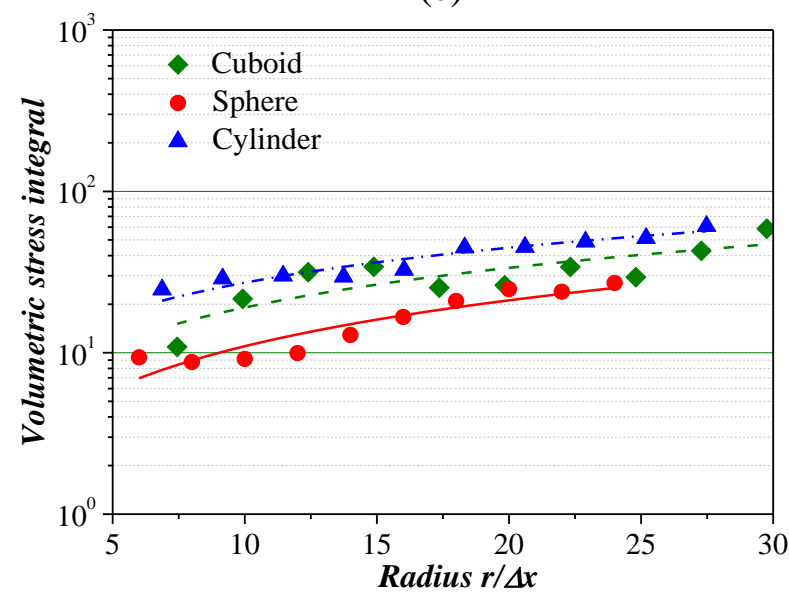

(e)

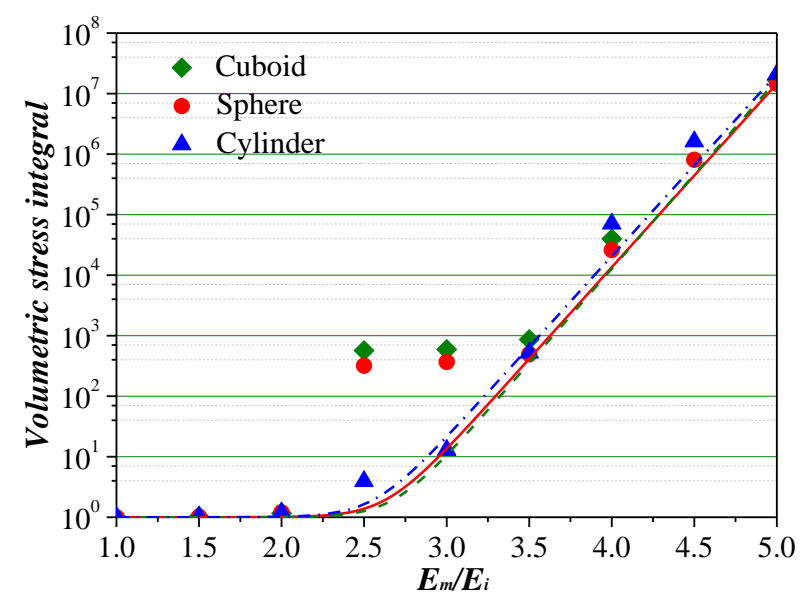

(b)

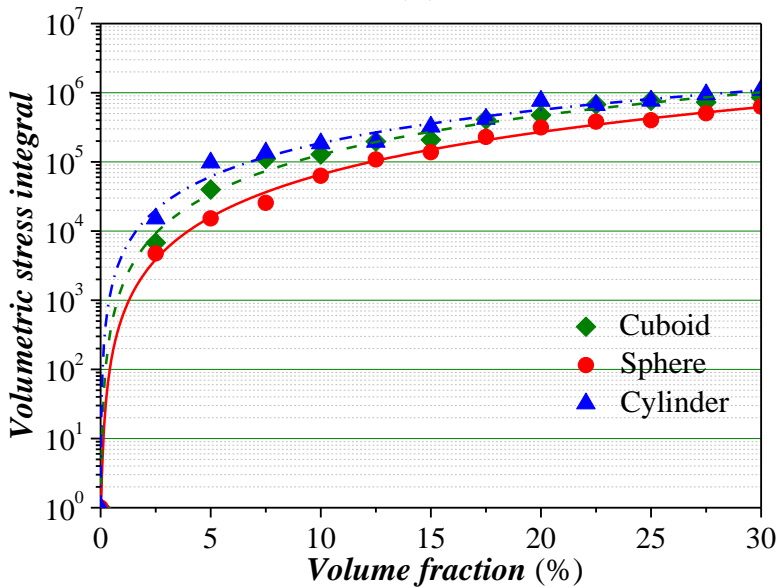

(d)

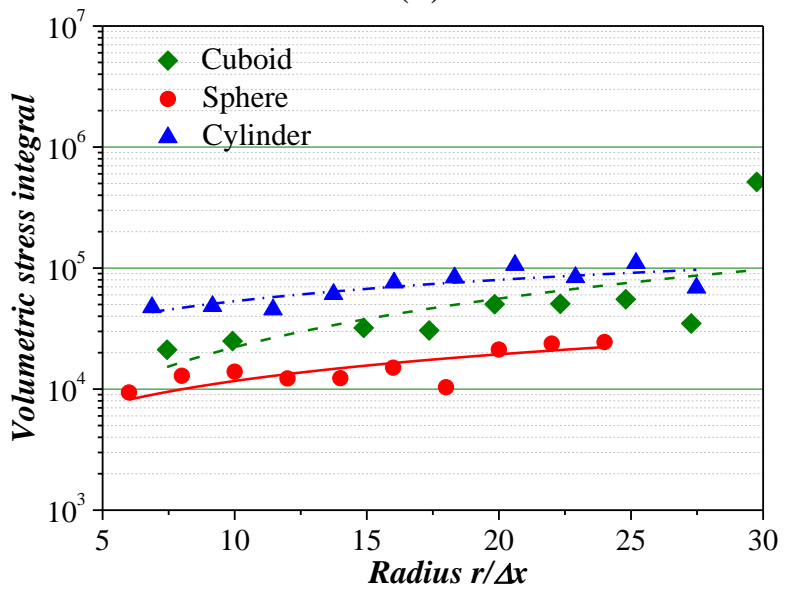

(f)

Figure 8: Comparisons among the volumetric stress integrals produced by inhomogeneities with sphere, cuboidal and cylinder shapes. (a) For stiff inhomogeneities of different material properties. (b) For compliant inhomogeneities of different material properties. (c) For stiff inhomogeneities of different volume fractions. (d) For compliant inhomogeneities of different volume fractions. (e) For stiff inhomogeneities of different sizes. (f) For compliant inhomogeneities of different sizes. 
Compared to spherical inhomogeneities, ellipsoidal inhomogeneities are more versatile. Various shapes can be simplified as ellipsoids conveniently. Therefore, the influence of ellipsoidal inhomogeneity on the stress field of material is compared with that of spherical inhomogeneity. Four kinds of ellipsoidal inhomogeneities, including two types of oblate ellipsoids and two types of prolate ellipsoids, are examined. Ellipsoid semi axes are represented as $r_{x}, r_{y}, r_{z}$. For oblate ellipsoids, set $r_{x}=r_{y}=2 r_{z}$ and $r_{x}=r_{y}=4 r_{z}$, respectively, while $r_{z}=2 r_{x}=2 r_{y}$ and $r_{z}=4 r_{x}=4 r_{y}$ for the other two types of prolate ellipsoids. The ellipsoids have the same volume with that of the corresponding spheres.

The variations of volumetric stress integrals with respect to material property, volume fraction and size are computed again for ellipsoidal inhomogeneities. The results, as illustrated in Fig. 9, reveal that the aspect ratio of ellipsoidal inhomogeneities has a significant influence on the stress field of contact body. Specifically, the integral deceases if spherical inhomogeneities are replaced by oblate ellipsoids. Prolate ones cause an increase in volumetric stress integral comparing to spheres, instead. Moreover, oblate inhomogeneities with a larger aspect ratio produce a smaller integral. On the contrary, an increase in the aspect ratio for prolate ellipsoids results in a larger integral value. The result also indicates that for ellipsoidal inhomogeneities, the orientation may affect the stress field of heterogeneous contact body. The RCF performance of heterogeneous material can be optimized through reasonable arrangement of distributed inhomogeneities if they are existed in materials inevitably. 


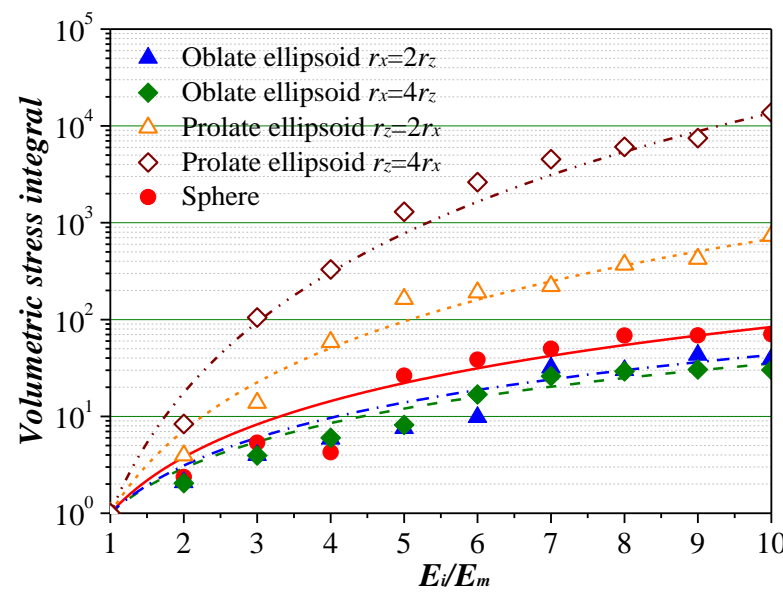

(a)

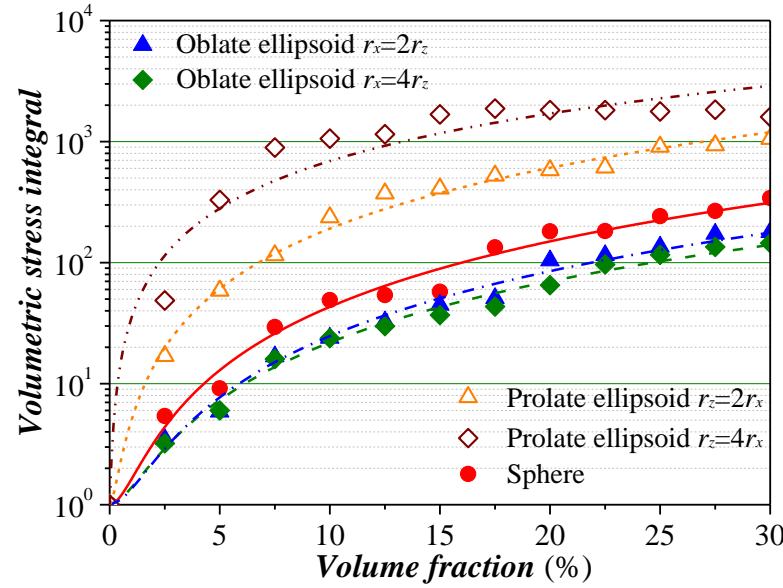

(c)

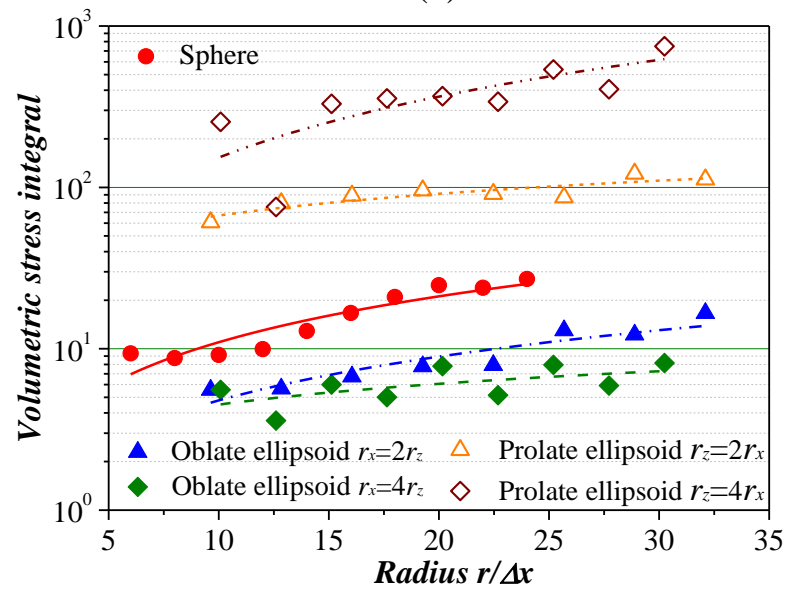

(e)

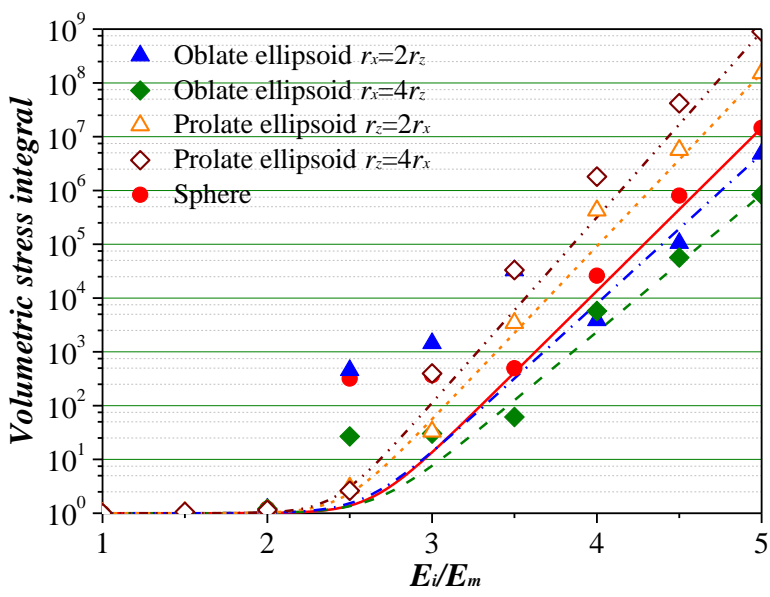

(b)

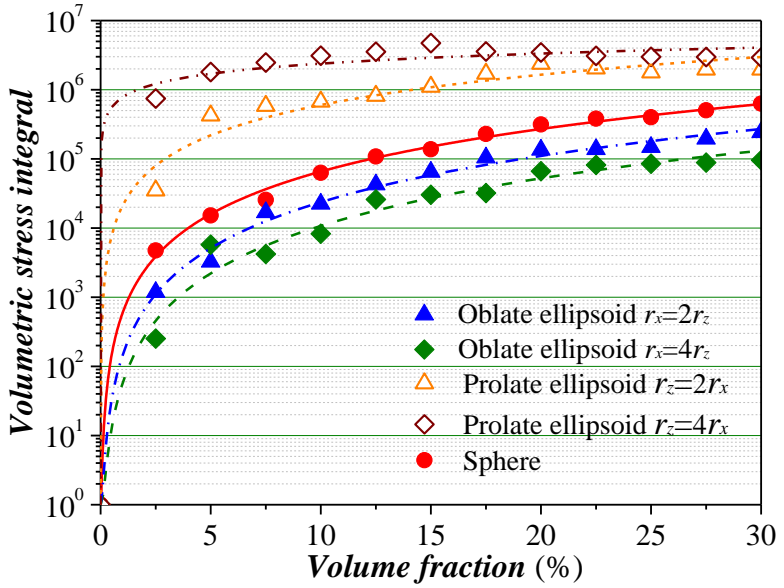

(d)

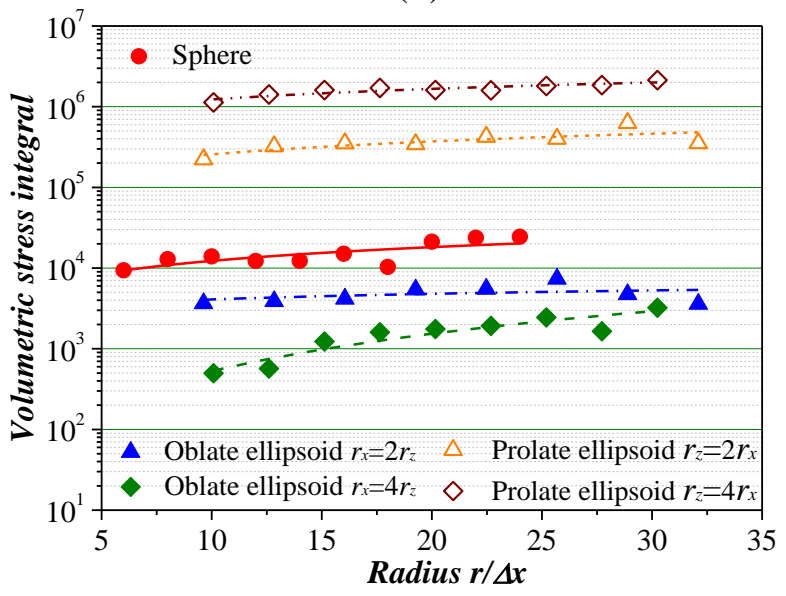

(f)

Figure 9: Comparisons among the volumetric stress integrals produced by ellipsoidal inhomogeneities with different aspect ratios. (a) For stiff inhomogeneities of different material properties. (b) For compliant inhomogeneities of different material properties. (c) For stiff inhomogeneities of different volume fractions. (d) For compliant inhomogeneities of different volume fractions. (e) For stiff 

sizes.

\subsection{Surface roughness}

Surface asperity is not considered in the above analyses. However, surface topography with different characteristics, such as those produced by different machining processes, is expected to have a significant influence on the RCF performance of a material. In this section, sinusoidal wavy surfaces, described by rms roughness and wavelength, are utilized. Sinusoidal wavy can be expressed as follows.

$$
z(x, y)=A \cdot \cos \left(2 \pi x / l_{w}\right) \cdot \cos \left(2 \pi y / l_{w}\right)
$$

where $A$ is the rms roughness, $l_{w}$ is the wavelength of sinusoidal wavy. Figure 10 a shows the sinusoidal roughness with $A$ fixed to $0.4 \mu \mathrm{m}$ and $l_{w}$ equals to $0.2 \mathrm{a}$ in both the $x$ and $y$ directions. If the sinusoidal wavy is added to the matrix model in Fig. 3a, different elastic fields will be obtained even if a same contact load is applied. The subsurface von Mises stress distribution of rough-surface contact with randomly distributed stiff $\left(E_{i} / E_{m}=4.0\right)$ cuboidal inhomogeneities is demonstrated in Fig. 10b. Surface asperities bring a remarkable effect on the stress field, especially for the domain near to the surface. For the present case, severer stress concentrations are observed for rough-surface heterogeneous contact. The relative maximum stress increases from 1.88 to 3.27 when a sinusoidal wavy surface is introduced. 


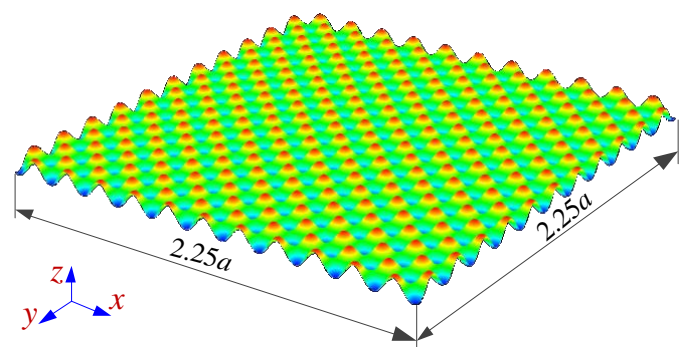

(a)

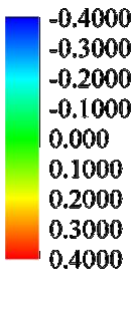

Figure 10: (a) A sinusoidal wavy surface. (b) von Mises stress field in the $x-z$ plane for roughsurface contact involving distributed stiff $\left(E_{i} / E_{m}=4.0\right)$ inhomogeneities.

The effective stressed volume for rough-surface heterogeneous contact is calculated. The $r m s$ roughness of the surface is changed from $0.2 \mu \mathrm{m}$ to $2.0 \mu \mathrm{m}$, while the wavelength is set to be $0.1 a$ and keeps unaltered. The effective depth $H_{e}$, as shown in Fig. 11, decreases rapidly with the increase in the surface rms roughness for both the stiff and compliant cases. For the case with stiff inhomogeneities, when the rms roughness exceeds $0.8 \mu \mathrm{m}$, the volumetric stress integrals of heterogeneous contact body is fully determined by surface topography. The corresponding critical rms roughness for the compliant case is $2.0 \mu \mathrm{m}$. Note that the descent rate of the effective depth for stiff case is larger than that for compliant case, as expected. The influence of surface topography on effective length $L_{e}$ is not such significant as that on effective depth, therefore the corresponding analysis result is not presented for brevity. 


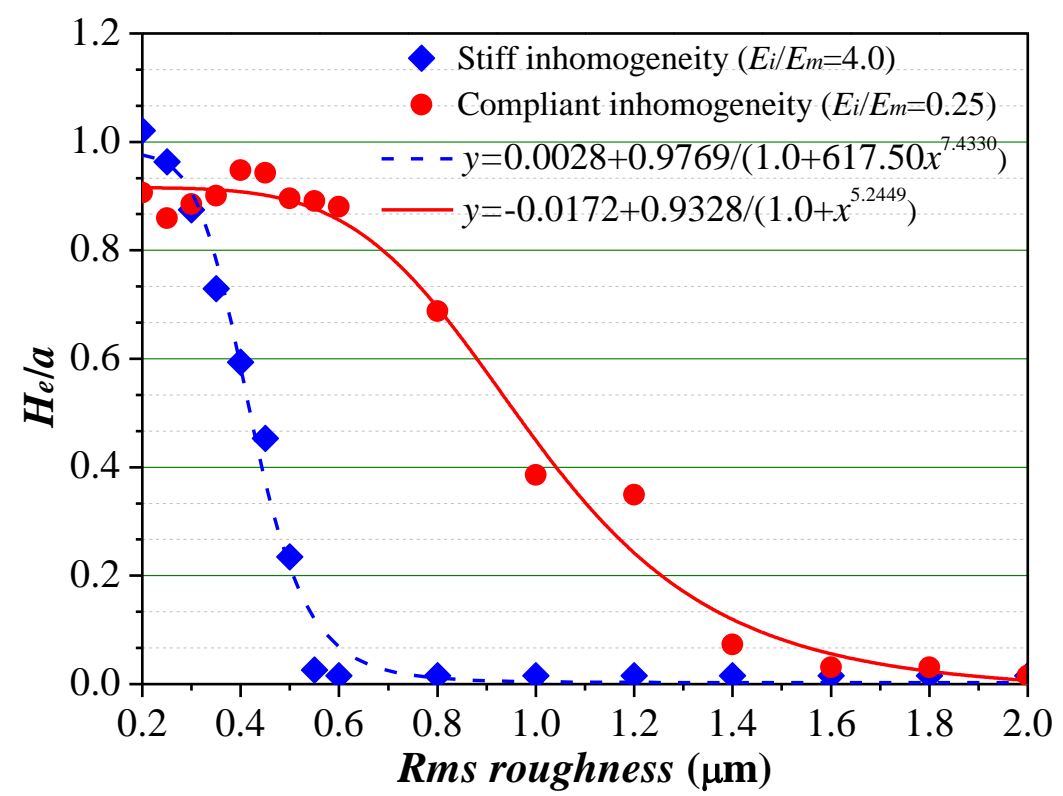

Figure 11: Effective depth for rough-surface contact involving stiff $\left(E_{i} / E_{m}=4.0\right)$ and compliant $\left(E_{i} / E_{m}=0.25\right)$ inhomogeneities

Parametric computations on rms roughness and wavelength are further conducted to investigate the influence of surface asperity characteristics on the volumetric stress integrals (RCF lives) of heterogeneous material. As illustrated in Fig. 12a, the wavelength $l_{w}$ is fixed to $0.1 a$, the $r m s$ roughness is varies from $0.2 \mu \mathrm{m}$ to $2.0 \mu \mathrm{m}$. Due to the consideration of surface roughness, the aggregate integral caused by both the surface asperity and inhomogeneity becomes larger. When rms roughness is small, distributed inhomogeneities determine the integral of the material. While rms roughness continues to grow, surface asperity starts to exert its influence and finally dominates the RCF performance of heterogeneous contact body. Asperity number located within the contact area can be controlled through the wavelength. The obtained volumetric stress integrals with the wavelength $l_{w}$ changing from $0.05 a$ to $0.5 a$ and $r m s$ roughness equals to $1.0 \mu \mathrm{m}$ are shown in Fig. 12b. It is concluded that more asperities within a fixed contact area may increase the integral of contact stressed volume, which is detrimental to the RCF lives of heterogeneous materials. 


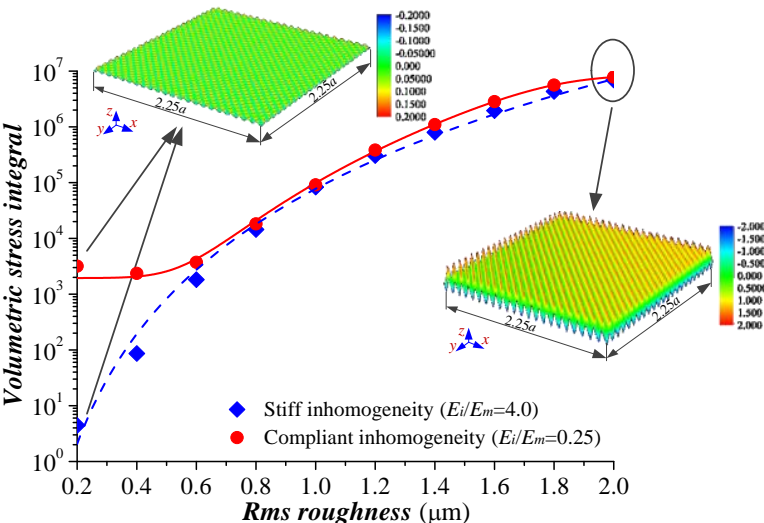

(a)

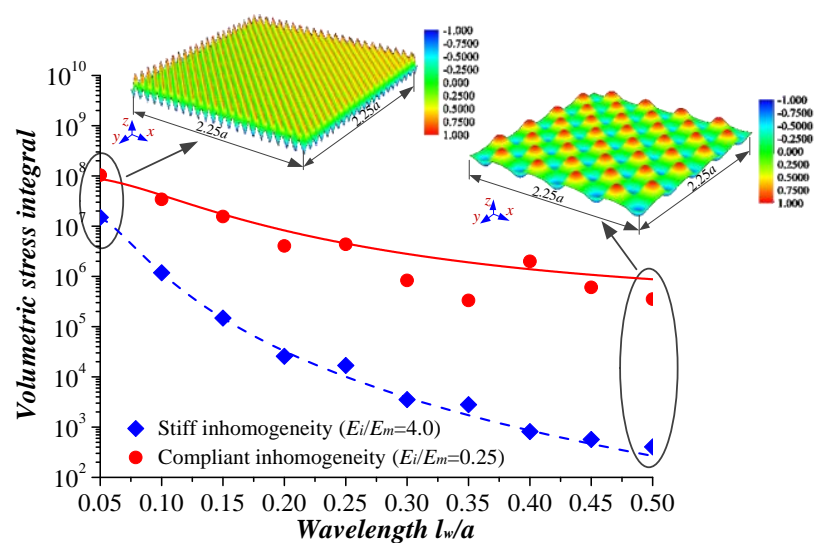

(b)

Figure 12: Volumetric stress integral variations of heterogeneous contact materials with sinusoidal wavy surfaces. (a) Influence of $r m s$ roughness with wavelength $l_{w}=0.1 a$. (b) Influence of wavelength with rms roughness $1.0 \mu \mathrm{m}$. 


\section{Conclusions}

The present study investigated the influence of distributed inhomogeneities and surface topography on the effective stressed volume of heterogeneous material subjected to contact loading. A recently developed numerical solution method was introduced to quantify the elastic field of rough-surface heterogeneous contact body. The solved effective stressed volume was then applied to conduct parametric studies on the influence of inhomogeneity and surface roughness on the volumetric stress integral of heterogeneous material. The following conclusions are obtained.

1) The effective stressed volume was achieved for a contact body distributed with inhomogeneities, which can be quantified by effective depth, length and width. Increases in the material difference, volume fraction and size of inhomogeneities shrink the effective stressed volume for both stiff and compliant cases.

2) Increases in the material difference, volume fraction and size of distributed inhomogeneities result in larger volumetric stress integrals for heterogeneous matrix. Compliant inhomogeneities produce severer stress concentrations within contact body, which are more detrimental to the RCF performance of inhomogeneous material than stiff ones.

3) Spherically shaped inhomogeneities yield smaller volumetric stress integrals than cuboidal and cylinder inhomogeneities. Ellipsoidal inhomogeneity and its aspect ratio have significant influences on the volumetric stress integral (RCF life) of a material.

4) Surface asperity adds more disturbances to the near-surface stress field of heterogeneous material, thus decreases the size of the effective stressed volume. Volumetric stress integral increases when rms roughness grows or more asperities are involved in contact area. However, 
further investigation on the coupled effect between surface asperity and subsurface inhomogeneity under contact loads is still necessary.

RCF life is one of the most important properties for mechanical components. The present work suggests that the domain outside of the effective stressed volume does not contribute to material RCF life. Near-surface particle and surface asperity dominate the RCF performance of heterogeneous contact body. The introduction of quantified stressed volume can reduce computational cost. More importantly, a smaller stressed volume provides a deeper insight into the RCF mechanism of heterogeneous contact. Engineering material is composed of matrix and second-phase materials with different material properties compared with the matrix. The computation results reveal that the distribution parameters, such as location, size, shape, etc., of second-phase materials have a significant influence on material RCF life. The present study can therefore be utilized as a theoretical guidance for material microstructure optimization to enhance the RCF performance of mechanical components.

\section{Acknowledgments}

The authors would like to acknowledge the support from National Natural Science Foundation of China (Grant nos. 51405316, 51435001) and the Center for Surface Engineering and Tribology at Northwestern University, USA. Q.Z. would like to acknowledge the supports from the Starting Foundation of Sichuan University (No. 2014SCU11062) and the Aeronautical Science Foundation of China (No. 20150219001). L.M.K would also like to acknowledge the support from US National Science Foundation (CMMI-1434834). 


\section{References}

[1] Sadeghi F, Jalalahmadi B, Slack TS, Raje N, Arakere NK. A Review of Rolling Contact Fatigue. ASME J Tribol. 2009;131:041403.

[2] Lundberg G, Palmgren A. Dynamic Capacity of Rolling Bearings. Acta Polytech Scand, Mech Eng Ser. 1947;1:1-52.

[3] Ioannides E, Harris T. A new fatigue life model for rolling bearings. ASME J Tribol. 1985;107:367-378.

[4] Zaretsky EY. Fatigue criterion to system design, life, and reliability. J Propul Power. 1987;3:76-83.

[5] Keer L, Bryant M. A pitting model for rolling contact fatigue. Journal of lubrication technology. 1983;105:198.

[6] Liu Y, Stratman B, Mahadevan S. Fatigue crack initiation life prediction of railroad wheels. Int J Fatigue. 2006;28:747-756.

[7] Weibull W. A statistical theory of the strength of materials, 1939. IVA Handlingar (Royal Swedish Academy of Engineering Sciences, Proceedings) nr. 1939;151.

[8] El-Thalji I, Jantunen E. A descriptive model of wear evolution in rolling bearings. Engineering Failure Analysis. 2014;45:204-224.

[9] Harris T, Yu WK. Lundberg-Palmgren fatigue theory: Considerations of failure stress and stressed volume. ASME J Tribol. 1999;121:85-89.

[10] Mura T. Micromechanics of Defects in Solids, 2nd ed. Dordrecht: Kluwer Academic; 1993.

[11] Eshelby JD. The Determination of the Elastic Field of an Ellipsoidal Inclusion, and Related Problems. Proc R Soc A. 1957;241:376-396.

[12] Eshelby JD. The Elastic Field Outside an Ellipsoidal Inclusion. Proc R Soc A. 1959;252:561-569.

[13] Shodja HM, Sarvestani AS. Elastic Fields in Double Inhomogeneity by the Equivalent Inclusion Method. ASME J Appl Mech. 2001;68:3.

[14] Benedikt B, Lewis M, Rangaswamy P. On elastic interactions between spherical inclusions by the equivalent inclusion method. Computational Materials Science. 2006;37:380-392.

[15] Jin X, Wang Z, Zhou Q, Keer LM, Wang Q. On the solution of an elliptical inhomogeneity in plane elasticity by the equivalent inclusion method. J Elast. 2014;114:1-18.

[16] Chiu YP. On the stress field due to initial strains in a cuboid surrounded by an infinite elastic space. ASME J Appl Mech. 1977;44:587-590.

[17] Zhou Q, Jin X, Wang Z, Wang J, Keer LM, Wang Q. Numerical implementation of the equivalent inclusion method for 2D arbitrarily shaped inhomogeneities. J Elast. 2015;118:39-61.

[18] Chiu YP. On the Stress Field and Surface Deformation in a Half Space With a Cuboidal Zone in Which Initial Strains Are Uniform. ASME J Appl Mech. 1978;45:302-306.

[19] Leroux J, Fulleringer B, Nélias D. Contact analysis in presence of spherical inhomogeneities within a half-space. Int J Solids Struct. 2010;47:3034-3049.

[20] Zhou Q, Jin X, Wang Z, Wang J, Keer LM, Wang Q. An efficient approximate numerical method for modeling contact of materials with distributed inhomogeneities. Int J Solids Struct. 2014;51:3410-3421.

[21] Zhou Q, Xie L, Jin X, Wang Z, Wang J, Keer LM, et al. Numerical Modeling of Distributed Inhomogeneities and Their Effect on Rolling Contact Fatigue Life. ASME J Tribol. 2015; 137:011402. 
[22] Wang Z, Jin X, Zhou Q, Ai X, Keer LM, Wang Q. An Efficient Numerical Method With a Parallel Computational Strategy for Solving Arbitrarily Shaped Inclusions in Elastoplastic Contact Problems. ASME J Tribol. 2013;135:031401.

[23] Liu S, Jin X, Wang Z, Keer LM, Wang Q. Analytical solution for elastic fields caused by eigenstrains in a half-space and numerical implementation based on FFT. International Journal of Plasticity. 2012;35:135-154.

[24] Zhou Q, Jin X, Wang Z, Wang J, Keer LM, Wang Q. Numerical EIM with 3D FFT for the contact with a smooth or rough surface involving complicated and distributed inhomogeneities. Tribol Int. 2016;93:91-103.

[25] Zhou Q, Jin X, Wang Z, Yang Y, Wang J, Keer LM, et al. A mesh differential refinement scheme for solving elastic fields of half-space inclusion problems. Tribol Int. 2016;93:124-136.

[26] Ai X. A Comprehensive Model for Assessing the Impact of Steel Cleanliness on Bearing Performance. ASME J Tribol. 2015, 137(1): 011101.

[27] Zhou Q, Xie L, Wang X, Jin X, Wang Z, Wang J, et al. Modeling rolling contact fatigue lives of composite materials based on the dual beam FIB/SEM technique. Int J Fatigue. 2016;83:201-208.

[28] Polonsky I, Keer L. A numerical method for solving rough contact problems based on the multi-level multi-summation and conjugate gradient techniques. Wear. 1999;231:206-219.

[29] Jacq C, Nélias D, Lormand G, Girodin D. Development of a Three-Dimensional SemiAnalytical Elastic-Plastic Contact Code. ASME J Tribol. 2002;124:653.

[30] Chen WW, Wang QJ. A numerical model for the point contact of dissimilar materials considering tangential tractions. Mech Mater. 2008;40:936-948.

[31] Liu S, Wang Q. Studying Contact Stress Fields Caused by Surface Tractions With a Discrete Convolution and Fast Fourier Transform Algorithm. ASME J Tribol. 2002;124:36.

[32] Dundurs J. Discussion“Edge-Bonded Dissimilar Orthogonal Elastic Wedges Under Normal and Shear Loading". ASME J Appl Mech. 1969;36:650-652.

[33] Wang Z, Jin X, Keer LM, Wang Q. Novel model for partial-slip contact involving a material with inhomogeneity. ASME J Tribol. 2013;135:041401.

[34] Greco A, Martini A, Liu Y, Lin C, Wang QJ. Rolling Contact Fatigue Performance of Vibro-Mechanical Textured Surfaces. Tribol Trans. 2010;53:610-620.

[35] Chapman SJ. Fortran 90/95 for scientists and engineers: McGraw-Hill Higher Education; 2004.

[36] Zaretsky EV. Rolling Bearing Life Prediction, Theory, and Application. Recent Developments in Wear Prevention, Friction and Lubrication. 2010:45-136. 